\title{
Expansion of mutation spectrum, determination of mutation cluster regions and predictive structural classification of SPAST mutations in hereditary spastic paraplegia
}

\author{
Moneef Shoukier ${ }^{1,2}$, Juergen Neesen ${ }^{1,2,3}$, Simone M Sauter ${ }^{1}$, Loukas Argyriou ${ }^{1}$, \\ Nadine Doerwald ${ }^{1}$, DV Krishna Pantakani ${ }^{1}$ and Ashraf U Mannan*,1 \\ ${ }^{1}$ Institute of Human Genetics, University of Goettingen, Goettingen, Germany
}

The SPAST gene encoding for spastin plays a central role in the genetically heterogeneous group of diseases termed hereditary spastic paraplegia (HSP). In this study, we attempted to expand and refine the genetic and phenotypic characteristics of SPAST associated HSP by examining a large cohort of HSP patients/families. Screening of 200 unrelated HSP cases for mutations in the SPAST gene led to detection of 57 mutations $(28.5 \%)$, of which 47 were distinct and 29 were novel mutations. The distribution analysis of known SPAST mutations over the structural domains of spastin led to the identification of several regions where the mutations were clustered. Mainly, the clustering was observed in the AAA (ATPases associated with diverse cellular activities) domain; however, significant clustering was also observed in the MIT (microtubule interacting and trafficking), MTBD (microtubule-binding domain) and an $\mathrm{N}$-terminal region (228-269 residues). Furthermore, we used a previously generated structural model of spastin as a framework to classify the missense mutations in the AAA domain from the HSP patients into different structural/functional groups. Our data also suggest a tentative genotype-phenotype correlation and indicate that the missense mutations could cause an earlier onset of the disease.

European Journal of Human Genetics (2009) 17, 187-194; doi:10.1038/ejhg.2008.147; published online 13 August 2008

Keywords: SPAST; spastin; HSP; mutation

\section{Introduction}

Hereditary spastic paraplegias (HSPs), also known as the Strümpell-Lorrain syndrome, are a group of neurodegenerative disorders caused by monogenic mutations. The universal clinical feature of the disease is progressive bilateral weakness and spasticity of the lower limbs. ${ }^{1}$

*Correspondence: $\mathrm{Dr}$ AU Mannan, Institute of Human Genetics, University of Goettingen, Heinrich-Dueker-Weg 12, D-37073, Goettingen, Germany. Tel: + 49551 397522; Fax + 49551 399303;

E-mail: amannan@gwdg.de

${ }^{2}$ These authors contributed equally to this work

${ }^{3}$ Current address: Department of Medical Genetics, Medical University of Vienna, Vienna, Austria

Received 15 January 2008; revised 9 July 2008; accepted 11 July 2008; published online 13 August 2008
Histopathological studies of HSP patients revealed selective degeneration of some of the longest axons. The degenerative process initially affects the distal ends of these axons and then proceeds proximally toward the cell body. $^{2,3}$ On the basis of clinical features, HSP can be classified into two forms: the pure and the complex HSP. In pure HSP, spasticity occurs in relative isolation; however, when additional neurological or non-neurological symptoms are associated with spasticity then it is termed as complex HSP. ${ }^{4,5}$

HSPs are genetically heterogeneous and different modes of inheritance are reported, including autosomal dominant, recessive and X-linked HSP; within each inheritance group, there is further locus heterogeneity. Fifteen of the 
HSP genes have been identified. However, mutations in the SPAST gene (MIM 604277) encoding for spastin protein is the most common cause of $\mathrm{HSP}^{6}$ and accounts for $15-40 \%$ of all AD-HSP cases, depending upon the ethnic origin of the selected cohort of patients. ${ }^{6-12}$ The prevalence of SPAST mutations in sporadic cases and with uncertain family history is much lower $(12-18 \%) .{ }^{13,14}$

Spastin belongs to the AAA (ATPases associated with diverse cellular activities) family of proteins. ${ }^{15-17}$ The AAA proteins are proven or putative ATPases and they are characterized by a conserved C-terminal domain containing one or two AAA cassettes. ${ }^{15}$ Apart from the AAA domain, these proteins consist of various other domains, which interact with adapter proteins to generate the structural and functional diversity of the family. ${ }^{16,18}$

Screening for mutations in the SPAST gene by various groups has identified over 224 different mutations in most exons except for exon 4, which is alternatively spliced. The SPAST mutations are summarized in the Human Gene Mutation Database Professional release 7.1 (http:// www.biobase.de/hgmd/pro/start.php). Different types of DNA alterations are detected in the SPAST gene, including missense, nonsense, splice site mutations and insertions/ deletions. Recent studies used a SPAST-specific multiplex ligation-dependent probe amplification (MLPA) assay to demonstrate that a large proportion (18-20\%) of patients with unlinked HSP, in fact, carry large deletions in the SPAST gene. ${ }^{19,20}$ These findings further broaden the spectrum of the SPAST mutations.

From a diagnostic point of view, unfortunately, there are no common mutations in SPAST, with most families having private mutations. Moreover, there are neither obvious genotype-phenotype correlations between different types of spastin mutations, nor any hot spot regions for SPAST mutations have been identified. Therefore, screening of the complete coding sequence of SPAST is necessary for the detection of mutations.

\section{Materials and methods HSP cases/families}

Blood samples from 200 unrelated HSP individuals/ families, primarily from Germany were referred to Goettingen Molecular Genetics Service for diagnostic testing of the SPAST gene. Clinical data of these patients were obtained by clinical evaluations from the referring neurologist. All probands were selected on the basis of Harding's accepted criteria for the definition of the clinical status of HSP. ${ }^{21}$ For ascertaining, a patient as HSP case following clinical features were assessed/observed in the lower limbs (lower extremities) of the patient; gait disorder owing to spastic paraparesis, spastic hypertonia, positive Babinski sign, ankle clonus, hyperreflexia, reduced pallesthesia and paresis. In addition, for complex HSP, cognitive deficits and other neurological/non-neurological symptoms were also considered. The age at onset (AAO) was determined by the referring neurologist after clinical evaluation and consulting with the patient. If required, additional family members were also consulted to corroborate the AAO. In our HSP cohort, 99 cases showed familial inheritance, 46 sporadic cases and in 55 cases, we could not ascertain the mode of inheritance. In this cohort, there were 109 pure HSP, 23 complex HSP and 68 unknown cases. Informed consent was obtained from all probands.

\section{Mutation screening and detection}

Genomic DNA from peripheral blood leukocytes of patients was isolated using standard procedures. The 17 exons of the SPAST gene (accession no. NM_014946.3) and flanking intronic sequences were amplified by PCR. Primer sequences and PCR conditions are available on request. Purification of PCR products was performed using a PCR purification kit (Millipore). The purified PCR products were sequenced using both forward and reverse primers (which were used for the PCR amplification) using ET reaction kit (Amersham Biosciences) on a MegaBACE 500 sequencer (Amersham Biosciences). Nucleotide variations revealed by first sequencing reaction were verified by second independent PCR and sequencing reaction. All sequence variants reported here were checked in a panel of 50 healthy unrelated subjects recruited randomly from the German population.

\section{MLPA analysis}

MLPA was performed with $200 \mathrm{ng}$ of genomic DNA according to manufacturer's instructions using the P165 Salsa MLPA HSP probe set (MRC-Holland). Probe amplification products were run on an ABI 3130 DNA Analyzer using Liz600 size standard (Applied Biosystems). MLPA peak plots were visualized, normalized and the dosage ratios were calculated by using GeneMarker Software v1.51 (Soft Genetics LLC). Owing to variation in each assay performance, we used dosage ratio values of $\leq 0.7$ and $\geq 1.35$ as our boundaries for deletions and duplications, respectively.

\section{Detection of break points for small insertions and deletions}

To determine the precise breakpoints of small insertions and deletions in the SPAST gene, the PCR amplicons were cloned into pGEMT Easy vector (Promega) according to the manufacturer's instructions. Plasmid DNA from at least 10 independent bacterial colonies were sequenced in both directions using vector-specific primers (T7 and SP6) using standard protocol.

\section{Modeling of the AAA domain of spastin}

The AAA ATPase domain of spastin was modeled on the basis of the tertiary structures of two templates (PDB codes: $1 \mathrm{xwi}$ and 1s3s) using MODELLER Version 8.0 program. $^{22}$ 
The generated models were energy-minimized using the Kollman united atom force field in SYBYL (Tripos Inc.) to ensure acceptable geometry and to relieve short contacts. The overall fit of the sequence to the template was checked using Verify 3D. ${ }^{22}$ The copies of modeled tertiary structures were assembled to form a hexameric quaternary assembly on the basis of the hexameric template (1s3s). This modeled quaternary structure was energy-minimized using SYBYL. ${ }^{22}$ The interfacial residues between the monomers were extracted using the Contacts of Structural Units program $^{22}$ and the crucial residues were short listed by manual inspection and used for further analysis.

\section{Labeling of mutated missense residues in the structural model of spastin}

We used a previously generated structural model of spastin, ${ }^{22}$ for labeling of the missense mutations identified in the AAA domain in our HSP cohort. In brief, the tertiary structure of the ATPase domain of spastin was modeled on the basis of the tertiary structures of two templates (PDB codes: 1xwi and 1s3s) and the quaternary structure was modeled on the basis of the hexameric template (1s3s). The consensus sequence/motifs in the AAA domain of spastin were structurally classified as active site, pore loop, protomer interacting and other residues and labeled as colored ribbons in the modeled tertiary structure. The novel spastin missense mutations identified in this study were labeled as colored space-fill structures in tertiary and quaternary structures using RasWin Molecular Graphics Version 2.7.3. (http://www.rasmol.org/).

The models of spastin are deposited in Protein Model Data Base (http://mi.caspur.it/PMDB/), which can be downloaded as PDB files (PDB codes: PM0074982 and PM0074984). The amino-acid residues in the spastin model are numbered from 1-286 aa, which corresponds to 331616 aa of the full-length spastin isoform. To determine in which functional/structural group (active site, pore loop structure, protomer interface residues and other mutations) of spastin a novel sequence variant can be cataloged, the mutated residue can be labeled by using RasWin Molecular Graphics Version 2.7.3 program.

\section{Results}

Detection of SPAST mutations in the HSP cases by sequencing and MLPA analysis

A total of 200 DNA samples of unrelated HSP individuals/ families were first screened for mutations in the SPAST gene by sequencing all the 17 exons of the gene, which led to the identification of $51(25.5 \%)$ heterozygous mutations (Table 1). Next, to detect SPAST deletion, we performed MLPA analysis in the 149 HSP cases, which were identified as SPAST negative through sequence analysis. By MLPA analysis, we identified six (three of which represented novel mutations) additional large heterozygous deletions in the SPAST gene, which accounts for 4\% of the 149 HSP cases (Table 1). In our screen, 47 different mutations were detected and 29 of these mutations were novel (Table 1). None of the 29 identified novel mutations in our study were found in the 100 control chromosomes. In the analyzed HSP cohort, the mutation rate in the SPAST gene in pure HSP was $29.4 \%(32 / 109)$, in complex HSP it was $21.7 \%(5 / 23)$ and in cases of unknown clinical course the proportion was $29.4 \%(20 / 68)$ (Table S1). Furthermore, in familial HSP we observed a mutation frequency of $36.4 \%$ (36/99), in sporadic HSP, it was 6.5\% (3/46) and in HSP cases with unknown inheritance pattern, we detected a mutation rate of $32.7 \%$ (18/55) ((Table S1), Note: Table S1S4 and Figure S1 are available as (online Supplementary Information)).

\section{Mutational spectrum and cluster regions in spastin}

The distribution analysis of the 26 novel mutations (excluding the gross deletions) along the structural domains of spastin revealed that $22(85 \%)$ mutations are localized in the conserved AAA domain, two (8\%) mutations were in the MIT domain (Figure 1a). Owing to this skewed spectrum of mutations in the spastin, we included other spastin mutations reported in the database (http:// www.biobase.de/hgmd/pro/start.php) in our analysis. Interestingly, we could identify several regions within the structural architecture of spastin where mutations were clustered (Figure 1b). The primary region was in the AAA domain (342-599 aa), which accounts for $72.7 \%$ of identified mutations in spastin (Figure $1 \mathrm{~b}$ and Table S2). Additional secondary cluster regions, were detected in the MIT domain (116-197 aa) with 7.2\% mutations, MTBD (microtubule-binding domain; $270-328$ aa) ${ }^{30}$ with $5.1 \%$ and an N-terminal region (228-269 residues) with $7.6 \%$ mutations (Figure $1 \mathrm{~b}$ and Table S2). Overall, these four regions account for $92.7 \%$ of the mutations reported to date in spastin.

\section{Functional/structural classification of the missense mutations}

Out of the 29 novel mutations, which we identified in our screen, 10 (38\%) are missense mutations, which are located in the AAA domain. We attempted to classify these 10 missense mutations identified in the AAA domain into different structural/functional groups based upon a previously generated structural model of spastin (PMBD id: PM0074982 and PM0074984). ${ }^{22}$ We could consign all these missense mutations in the three dimensional space of the spastin structure into four categories namely, active site, pore loop structure, protomer interface residues and other mutations (Table S3 and Figure S1A-K).

\section{S44L polymorphism in HSP}

Co-inheritance of a disease-causing mutation and the L44 allele causes an early onset of symptoms, indicating that 
Table 1 List of mutations identified in the SPAST gene in our HSP cohort

\begin{tabular}{|c|c|c|c|c|c|c|c|c|c|}
\hline No. & Exon/Intron & Family no. & Type of mutation & $c D N A$ & Protein & $F S$ & $\begin{array}{l}\text { Onset of } \\
\text { phenotype }\end{array}$ & Pure/complex & $\begin{array}{c}\text { Referencel } \\
\text { novel }\end{array}$ \\
\hline 1 & Ex 1 & 24227 & Nonsense & c. $373 G>T$ & p.Glu125Term & $\mathrm{F}$ & $>35$ & Pure & 11 \\
\hline 2 & Ex 2 & 25942 & Nonsense & c. $499 \mathrm{C}>\mathrm{T}$ & p.Gln167Term & $S$ & $>35$ & Pure & Novel \\
\hline 3 & Ex 3 & 24283 & Insertion & c.549_550insT & p.Asn184Term & $\mathrm{F}$ & $<35$ & Pure & Novel \\
\hline 4 & Ex 5 & 21987 & Deletion & c.692delC & $\begin{array}{l}\text { p.Ala231 Valfs } \\
\text { Term239 }\end{array}$ & $?$ & $?$ & $?$ & Novel \\
\hline 5 & Ex 5 & 19583 & Deletion & c.839_840delAG & $\begin{array}{l}\text { p.GIn280Argfs } \\
\text { Term } 289\end{array}$ & $\mathrm{~F}$ & $<35$ & Pure & 12 \\
\hline 6 & Ex 5 & 28146 & Splice & c. $870 \mathrm{G}>\mathrm{A}$ & Unknown & $?$ & $?$ & $?$ & Novel \\
\hline 7 & Int 5 & 25961 & Splice & c. $870+1 \mathrm{G}>\mathrm{T}$ & Unknown & $?$ & $?$ & $?$ & Novel \\
\hline 8 & Ex 6 & 24295 & Insertion & c.981_982insAT & p.lle328Ilefs & $\mathrm{F}$ & $<35$ & $\begin{array}{c}\text { Complicated } \\
\text { (cardio-myopathy) }\end{array}$ & Novel \\
\hline 9 & Ex 7 & 21937 & Missense & c. $1067 A>G$ & p.Glu356Gly & $?$ & $<10$ & ? & 6 \\
\hline 10 & Ex 7 & 21935 & Missense & c. $1081 \mathrm{C}>\mathrm{T}$ & p.Pro361Ser & $\mathrm{F}$ & $<35$ & Pure & Novel \\
\hline 11 & Int 7 & 21971 & Splice/deletion & c.1099-3_1099-1delTAG & Unknown & $\mathrm{F}$ & $>35$ & Pure & Novel \\
\hline 12 & Ex 8 & 21977 & Deletion & c.1101_1103delGTT & p.Leu367del & $\mathrm{F}$ & $>35$ & Pure & Novel \\
\hline 13 & Ex 8 & 25902 & Deletion & c.1101_1103delGTT & p.Leu367del & $\mathrm{F}$ & $<10$ & Pure & Novel (2) \\
\hline 14 & Ex 8 & 24218 & Insertion & c.1115_1116insG & $\begin{array}{l}\text { p.Arg372Argfs } \\
\text { Term393 }\end{array}$ & $?$ & $?$ & $?$ & Novel \\
\hline 15 & Ex 8 & 25945 & Missense & c. $1121 \mathrm{C}>\mathrm{G}$ & p.Pro374Arg & $\mathrm{F}$ & $<10$ & $?$ & Novel \\
\hline 16 & Ex 8 & 25946 & Missense & c. $1154 \mathrm{G}>\mathrm{A}$ & p.Gly385Glu & $S$ & $>35$ & Pure & Novel \\
\hline 17 & Int 8 & 24292 & Splice & c. $1174-1 G>T$ & Unknown & $\mathrm{F}$ & $<35$ & Pure & Novel \\
\hline 18 & Ex 9 & 21938 & Missense & c. $1196 \mathrm{C}>\mathrm{T}$ & p.Ser399Leu & $\mathrm{F}$ & $?$ & $?$ & 23 \\
\hline 19 & Ex 9 & 24224 & Missense & c. $1196 \mathrm{C}>\mathrm{T}$ & p.Ser399Leu & $?$ & ? & $?$ & 23 \\
\hline 20 & Ex 9 & 19576 & Deletion & c. $1202 \mathrm{delC}$ & $\begin{array}{l}\text { p.Ala401Gluf- } \\
\text { sTerm406 }\end{array}$ & $?$ & $?$ & $?$ & Novel \\
\hline 21 & Ex 9 & 24286 & Deletion & c.1215_1219delTATAA & $\begin{array}{l}\text { p.Asn } 405 \text { Lysf- } \\
\text { sTerm } 440\end{array}$ & $S$ & $>35$ & Pure & 8 \\
\hline 22 & Ex 9 & 24268 & Missense & c. $1216 \mathrm{~A}>\mathrm{G}$ & p.lle406Val & $\mathrm{F}$ & $<35$ & Pure & 24 \\
\hline 23 & Ex 9 & 21901 & Deletion & c. 1245 delC & p.Tyr415Term & $S$ & $>35$ & Pure & Novel \\
\hline 24 & Int 9 & 24248 & Splice & c. $1245+1 G>T$ & Unknown & $\mathrm{F}$ & $<10$ & Pure & 25 \\
\hline 25 & Ex 10 & 24231 & Missense & c. $1250 \mathrm{G}>\mathrm{A}$ & p.Gly417Glu & $\mathrm{F}$ & $<35$ & $\begin{array}{l}\text { Complicated } \\
\text { (cognitive } \\
\text { impairment) }\end{array}$ & Novel \\
\hline 26 & Ex 10 & 19582 & Missense & c. $1280 \mathrm{~T}>\mathrm{G}$ & p.Phe427Cys & $\mathrm{F}$ & $>35$ & Pure & Novel \\
\hline 27 & Ex 10 & 24233 & Deletion & c. $1281 \mathrm{delT}$ & $\begin{array}{l}\text { p.Phe427Leufs } \\
\text { Term } 437\end{array}$ & $?$ & $?$ & $?$ & 26 \\
\hline 28 & Ex 10 & 24212 & Nonsense & c. $1291 \mathrm{C}>\mathrm{T}$ & p.Arg431Term & $\mathrm{F}$ & $>35$ & Pure & 8 \\
\hline 29 & Ex 11 & 19593 & Missense & c. $1339 \mathrm{~T}>\mathrm{G}$ & p.Leu447Val & $\mathrm{F}$ & $>35$ & Pure & Novel \\
\hline 30 & Ex 11 & 21214 & Missense & c. $1378 . C>T$ & p.Arg460Cys & $\mathrm{F}$ & $>35$ & $\begin{array}{c}\text { Complicated } \\
\text { (polyneuro-pathay) }\end{array}$ & 38 \\
\hline 31 & Ex 11 & 24222 & Missense & c. $1378 \mathrm{C}>\mathrm{T}$ & p.Arg460Cys & $?$ & $<10$ & $?$ & \\
\hline 32 & Ex 11 & 24285 & Missense & c. $1378 \mathrm{C}>\mathrm{T}$ & p.R460C & $\mathrm{F}$ & $<35$ & Pure & 27 \\
\hline 33 & Ex 11 & 24228 & Missense & c. $1379 \mathrm{G}>\mathrm{A}$ & p.Arg460Cys & $\mathrm{F}$ & $<35$ & Pure & Novel \\
\hline 34 & Ex 12 & 25910 & Nonsense & c. $1417 \mathrm{C}>\mathrm{T}$ & p.Arg $460 \mathrm{His}$ & $\mathrm{F}$ & $<10$ & $\begin{array}{l}\text { Autonomic nervous } \\
\text { system }\end{array}$ & Novel \\
\hline 35 & Ex 12 & 24255 & Insertion & c.1462_1463insTA & p.GIn473Term & $\mathrm{F}$ & $>35$ & Pure & Novel \\
\hline 36 & Ex 13 & 25923 & Missense & c. $1495 \bar{C}>\mathrm{T}$ & $\begin{array}{l}\text { p.Arg488llef- } \\
\text { sTerm530 }\end{array}$ & $\mathrm{F}$ & $<10$ & Pure & \\
\hline 37 & Ex 13 & 21929 & Missense & c. $1496 \mathrm{G}>\mathrm{A}$ & p.Arg499Cys & $\mathrm{F}$ & $<10$ & Trunk-ataxia & 28 \\
\hline 38 & Ex 13 & 19598 & Missense & c. $1507 \mathrm{C}>\mathrm{T}$ & p.Arg $499 \mathrm{His}$ & $?$ & $?$ & ? & 14 \\
\hline 49 & Ex 14 & 21900 & Missense & c. $1540 A>G$ & p.Arg503Trp & $\mathrm{F}$ & $?$ & $?$ & Novel \\
\hline 40 & Ex 14 & 21985 & Missense & c. $1540 A>G$ & p.Arg514Gly & $\mathrm{F}$ & $>35$ & $?$ & Novel (2) \\
\hline 41 & Ex 15 & 24230 & Insertion & c.1649_1650insCCTAAC & p.Arg514Gly & $\mathrm{F}$ & $<35$ & Pure & Novel \\
\hline 42 & Ex 15 & 19591 & Missense & c. $1664 \bar{A}>G$ & $\begin{array}{l}\text { p.550_551in- } \\
\text { sLeuThr }\end{array}$ & $\mathrm{F}$ & $<35$ & Pure & Novel \\
\hline 43 & Ex 15 & 25941 & Missense & c. $1670 \mathrm{C}>\mathrm{T}$ & p.Asp555Gly & $\mathrm{F}$ & $<35$ & Pure & Novel \\
\hline 44 & Ex 15 & 21920 & Nonsense & c. $1684 \mathrm{C}>\mathrm{T}$ & p.Ala557Val & $?$ & $?$ & $?$ & \\
\hline 45 & Ex 15 & 21967 & Nonsense & c. $1684 \mathrm{C}>\mathrm{T}$ & p.Arg562Term & $?$ & $>35$ & Pure & 8 \\
\hline 46 & Ex 15 & 21974 & Nonsense & c. $1684 \mathrm{C}>\mathrm{T}$ & p.Arg562Term & $\mathrm{F}$ & $>35$ & Pure & 8 \\
\hline 47 & Ex 15 & 24201 & Nonsense & c. $1684 \mathrm{C}>\mathrm{T}$ & p.Arg562Term & $\mathrm{F}$ & $<35$ & Pure & 8 \\
\hline 48 & Ex 15 & 25912 & Nonsense & c. $1684 \mathrm{C}>\mathrm{T}$ & p.Arg562Term & $\mathrm{F}$ & $>35$ & Pure & 8 \\
\hline 49 & Ex 16 & 19594 & Nonsense & c. $1702 \mathrm{C}>\mathrm{T}$ & p.Arg562Term & $?$ & $?$ & $?$ & Novel \\
\hline 50 & Ex 17 & 19597 & Missense & c. $1821 G>C$ & p.GIn568Term & ? & ? & ? & 13 \\
\hline 51 & Ex 17 & 25936 & Missense & c. $1821 G>C$ & p.Trp607Cys & $\dot{\mathrm{F}}$ & $<35$ & Pure & 13 \\
\hline 52 & Ex 1- Ex 3 & 24278 & Exon deletion & c. $1-? \_682+? \mathrm{del}$ & Unknown & $?$ & $?$ & $?$ & 19 \\
\hline 53 & Ex 2- Ex 9 & 21976 & Exon deletion & c.416-?_1493+?del & Unknown & $\mathrm{F}$ & $<35$ & Pure & Novel \\
\hline 54 & Ex 8 & 21968 & Exon deletion & c.1099-?_1173+?del & Unknown & $\mathrm{F}$ & $<10$ & Pure & Novel \\
\hline 55 & Ex $9-$ Ex 17 & 21940 & Exon deletion & c. $1174-?-1851+? \mathrm{del}$ & Unknown & $\mathrm{F}$ & $>35$ & Pure & Novel \\
\hline 56 & Ex $2-$ Ex16 & 24270 & Exon deletion & c.416-?_1728+?del & Unknown & $\mathrm{F}$ & $>35$ & Pure & 29 \\
\hline 57 & Ex 17 & 24281 & Exon deletion & c.1729-?_18511?del & Unknown & $?$ & $?$ & $?$ & 19 \\
\hline
\end{tabular}

Note: F, Familial; S, Sporadic. 
a

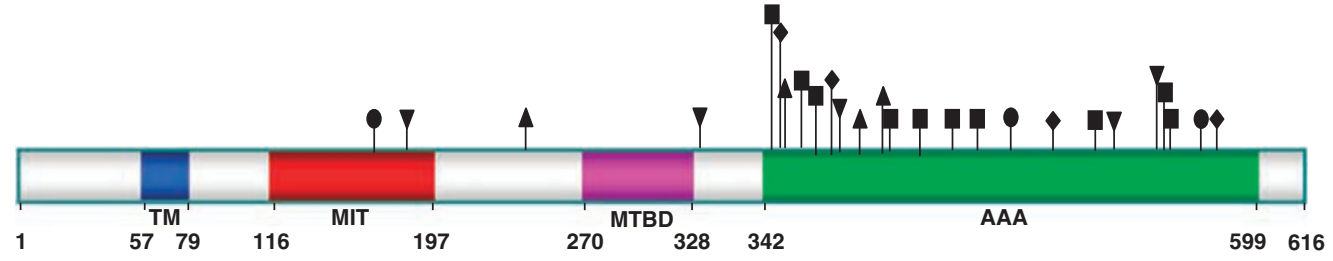

b

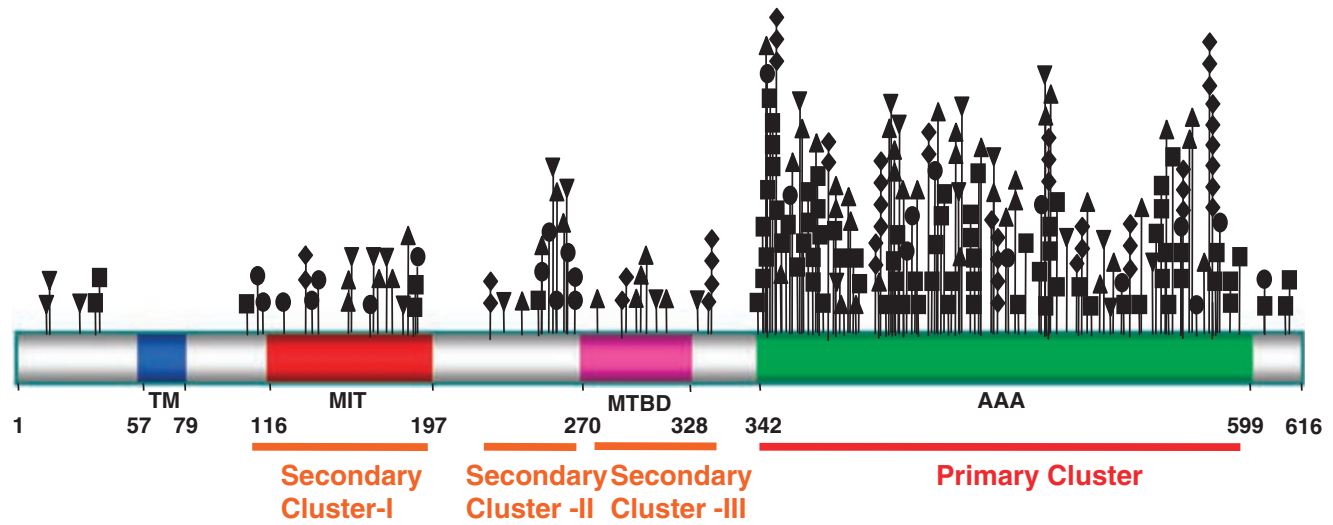

- Missense $\quad$ Nonsense $\bullet$ Splice site $\quad$ Insertion $\quad$ Deletion

TM - Transmembrane Domain; MIT - Microtubule Interacting and Trafficking Domain

MTBD - Microtubule Binding Domain; AAA - ATPase Associated with Various Cellular Activities Domain

Figure 1 Schematic diagram representing the structural domains of the spastin protein (616 amino acids) and showing the localization of the identified mutations. The different domains are highlighted with different colors and different kinds of mutations are marked with different symbols as summarized on the left at the bottom of the figure. (a) Representation of 26 novel mutations identified in our study. Twenty-two out of 26 mutations are located in the AAA domain and two others in the MIT domain. (b) Distribution of all mutations identified to date in the spastin protein. The primary hot spot region is indicated as a red bar in the AAA domain. Three secondary hot spot regions are shown as orange bars below the spastin structure. Note: Three novel gross deletions are not included for representation; also gross deletions are excluded for distribution analysis.

the L44 allele is a genetic modifier of the HSP disease. ${ }^{10,31}$ In our cohort of 200 HSP patients, the S44L variant was identified in six unrelated HSP cases in heterozygous state, therefore the incidence of the L44 allele in our cohort is $1.5 \%$. In addition, in two HSP individuals, we detected a second mutation in SPAST apart from the L44 allele (Table S4).

\section{Putative genotype-phenotype correlations}

To determine whether the spastin missense mutations have a different pathomechanism in contrast to other mutation types is to examine a correlation between mutation types and clinical features, such as age at onset of the symptoms. We therefore grouped the 57 identified mutations in our HSP cohort into two groups: the missense mutations (23) and the other types of mutations (34). We placed the number of identified mutations into two categories based upon age at onset of the symptoms, namely, onset before/ at 35 years $(\leq 35)$ and after 35 years $(>35)$ as represented in
Table 2 The proportions of missense and other types of mutations in different age groups, pure/complex and familial/sporadic cases of HSP

\begin{tabular}{lccc}
\hline & Missense mutations & Other mutations & All mutations \\
\hline & 23 & 34 & 57 \\
$<35$ years & 13 & 13 & 26 \\
$>35$ years & 3 & 10 & 13 \\
Unknown & 7 & 11 & 18 \\
& & & \\
Pure & 11 & 21 & 32 \\
Complex & 3 & 2 & 5 \\
Unknown & 9 & 11 & 20 \\
& & 20 & 36 \\
Familial & 16 & 2 & 3 \\
Sporadic & 1 & 12 & 18 \\
Unknown & 6 & &
\end{tabular}

Table 2 . In the $\leq 35$ age group, we observed an equal proportion of missense mutations $(13 / 26 ; 50 \%)$ and other types of mutations $(13 / 26 ; 50 \%)$ (Table 2$)$. In the $>35$ age 
group, we detected a higher fraction of other mutation types, $(10 / 13 ; 76.9 \%)$ as compared with missense mutations $(3 / 13 ; 23 \%)$ (Table 2$)$. However, statistically the differences between the proportion of missense and other mutations in $>35$ age group was not significant $(P>0.05)$. Interestingly, we noticed a skewed distribution of missense mutations between the two age groups; $\leq 35$ and $>35$ years. Therefore, we tested a null hypothesis that there is no difference in the proportion of the subjects with missense mutations between two age groups $\leq 35$ and $>35$ years, the chi-square test demonstrated that there is a significant difference $(P<0.012)$, whereas no significant difference could be detected for subjects with other mutation types $(P>0.5)$.

\section{Discussion}

In our current endeavor, we attempted to expand the mutational spectrum of SPAST. To this end, we screened for mutations in the SPAST gene in 200 HSP patients and identified 47 different mutations, out of which 29 were novel mutations. The overall frequency of SPAST mutations in our cohort was $28.5 \%(57 / 200)$. The mutation rate did not change significantly when we only considered pure HSP, which was $29.4 \%(32 / 109)$. Interestingly, in case of complex HSP a high mutational rate of $21.7 \%(5 / 23)$ was detected in our HSP cohort, which highlights the need to screen for the SPAST mutation in complex HSP cases. However, owing to lack of family history, we were unable to show segregation of additional symptom(s) with paraplegia, therefore, it is also possible that the complex phenotype could also be because of an independent locus other than the SPAST. The mutation detection rate in our cohort is consistent with the range of $15-44 \%$, which was observed previously in other populations. ${ }^{8-11,13}$ The frequency of mutations increased if we only considered the autosomal dominant HSP cases (36.4\%) suggesting that prevalence of SPAST mutation is higher in the familial cases. Among the sporadic cases of HSP, the frequency of mutations was $6.5 \%(3 / 46)$, which was lower than the previously reported rate of $12-18 \%{ }^{13,14}$ This discrepancy could be because of the different population type and size. Nevertheless, from a diagnostic point of view, our and other reports emphasize the need to screen for SPAST mutations in the sporadic HSP cases.

In our HSP cohort, we identified 51 mutations in the SPAST gene by direct sequencing of all the 200 HSP patients, which left 149 HSP cases in which no mutations could be detected by conventional sequencing. To determine gross deletion/insertion in the SPAST gene, we performed MLPA analysis in these 149 HSP cases and detected six additional mutations, which accounts for $4 \%$ $(6 / 149)$ of remaining HSP cases. Previously, two independent studies used same SPAST-specific MLPA assay and reported a much higher proportion (18-20\%) of deletion in HSP patients. ${ }^{19,20}$ The observed disparity in the proportion of large deletions between our HSP cohort and others could be because of the divergence and ethnic variability in these cohorts. Nevertheless, our report of much lower proportion of gross deletions in the SPAST gene in the HSP patients stresses the need to perform MLPA in various HSP cohorts to determine the incidence rate of gross deletions in worldwide HSP populations.

It is remarkable that $22(85 \%)$ out of the 26 novel mutations (excluding the gross deletions) were located in the AAA domain of spastin. Previously, our group reported clustering of mutations in the AAA domain of spastin in a German HSP cohort $^{12}$ and this clustering in AAA domain was also observed in several other HSP cohorts. ${ }^{6-10}$ Moreover, the distribution of mutations reported in the database over the structural domains of spastin outside the AAA domain were also not uniform; rather they were concentrated in certain regions of the protein, which constituted various functional domains, such as MIT and MTBD. In prior studies, exon 1, exon 5 and exon 8 of SPAST was recognized as hot spot regions; $;^{32-34}$ however, no correlation to functional domain of spastin was implicated. Overall, it appears that different functional domains of spastin are target regions for mutations, which underlines their functional significance. Identification of these cluster regions highlights the need to set these regions as priority in the molecular diagnostic screens.

Beside a few exceptions, almost all the missense mutations in spastin are located in the AAA domain and recent studies suggest that these missense mutations might exert a dominant-negative effect on the molecular function of spastin. ${ }^{35,36}$ Utilization of a recently modeled structure of the AAA domain of spastin, ${ }^{22}$ as a framework, enabled us to classify the identified missense mutations from our cohort into different functional groups such as active site, protomer-protomer interaction, pore loop and unknown structural group of mutations. The functional categorization of the novel missense mutations, based upon the structural model of spastin will enable us in future to predict any identified sequence variant in a HSPSPAST patient as disease-causing mutation with greater level of certainty. These structural predictions of various functional classes of missense mutations need to be validated by biochemical/cellular studies and data from the structural model should be interpreted with cautiousness. However, in a recent study, we could validate at the cellular level the functional effect of two sequence variants (E442Q and R499C) of spastin, which were predicted as active site mutations from the structural model of spastin. ${ }^{22}$

The rare $\mathrm{S} 44 \mathrm{~L}$ polymorphism is considered to act as a modifier of the HSP phenotype. ${ }^{10,31,37}$ S44L is not considered as a susceptibility factor for HSP because its 
frequency rate is similar in HSP patients and controls. ${ }^{14}$ In our study, we could not ascertain the role of S44L (heterozygous state alone) on manifestation of HSP. It is possible that the patients heterozygous for S44L might have another mutation in spastin, which could not be identified by our screen or might have a mutation in a different HSP gene.

No apparent genotype-phenotype correlation is evident among missense mutations and other SPAST mutations. ${ }^{8,14,38}$ Although several studies indicated that missense mutation might act in a dominant-negative fashion in contrast to other mutations, which lead to a loss of function. To determine, whether missense mutation leads to early onset of HSP, we assorted our HSP cohort into two different groups based upon AAO ( $\leq 35$ and $>35$ years). The rational behind sorting our HSP cohort into these two age groups was derived from Harding's classification of HSP patients into two distinct groups, early age onset $(\leq 35$ years) and late age onset ( $>35$ years). ${ }^{39}$ This AAO ( $\leq 35$ and $>35$ years) classification was also used by Fonknechten and coworkers for determination of genotype-phenotype correlation. ${ }^{8}$ We observed an obvious difference in the proportion of mutations between the missense group as compared with the other types of mutations in age group of $>35$ years. However, the observed difference was not statistically significant $(P>0.05)$ because of a very small sample size. Remarkably, we could reject a null hypothesis that there is no difference in the proportion of subjects for missense mutations between two age groups, $\leq 35$ and $>35$ years, which was statistically significant $(P<0.0124)$. Our data show a tentative genotype-phenotype correlation and suggest that in case of missense mutations the onset of phenotype is earlier. Owing to a small sample size, this correlation between $\mathrm{AAO}$ and missense mutation should be interpreted with discretion. Previously, early $\mathrm{AAO}$ in patients with missense mutation was also reported; however, this study only accounted for two missense mutations out of a total five mutations. ${ }^{40}$ Moreover, in a meta-analysis $^{38}$ no significant correlation between AAO and mutational class was evident, but one limitation of this study was the sample size. Nevertheless, these different pathomechanism modes, such as loss of function and dominant-negative function for different classes/types of spastin mutations need to be carefully resolved by experimental means; otherwise there will be repercussions on the likely success of any therapeutical approach devised for spastin-associated HSP.

\section{Acknowledgements}

We thank the HSP patients/families for their participation in this study, B Brandt for excellent technical assistance, P Grzmil for statistical analysis and $W$ Engel for critical comments/reading of the paper. This work was funded partly by a Tom Wahlig Stiftung Grant and the Deutsche Forschungsgemeinschaft (MA 3344/2-1) Grant to $A U M$ and by the institution's internal fund.

\section{References}

1 Bruyn RPM, Scheltens P: Hereditary spastic paraparesis (Strümpell-Lorrain), Diseases of the motor system; in de Jong JMBV (ed): Handbook of Clinical Neurology. Elsevier Science Publishers: Oxford, 1991, vol 15, pp 301-318.

2 Behan WM, Maia M: Strumpell's familial spastic paraplegia: genetics and neuropathology. J Neurol Neurosurg Psychiatry 1974; 37: 8-20.

3 Schwarz GA, Liu CN: Hereditary (familial) spastic paraplegia; further clinical and pathologic observations. AMA Arch Neurol Psychiatry 1956; 75: 144-162.

4 Fink JK: The hereditary spastic paraplegias: nine genes and counting. Arch Neurol 2003; 60: 1045-1049.

5 Reid E: Science in motion: common molecular pathological themes emerge in the hereditary spastic paraplegias. J Med Genet 2003; 40: 81-86.

6 Hazan J, Fonknechten N, Mavel D et al: Spastin, a new AAA protein, is altered in the most frequent form of autosomal dominant spastic paraplegia. Nat Genet 1999; 23: 296-303.

7 McDermott CJ, Burness CE, Kirby J et al: Clinical features of hereditary spastic paraplegia due to spastin mutation. Neurology 2006; 67: 45-51.

8 Fonknechten N, Mavel D, Byrne P et al: Spectrum of SPG4 mutations in autosomal dominant spastic paraplegia. Hum Mol Genet 2000; 9: 637-644.

9 Hentati A, Deng HX, Zhai $\mathrm{H}$ et al: Novel mutations in spastin gene and absence of correlation with age at onset of symptoms. Neurology 2000; 55: 1388-1390.

10 Lindsey JC, Lusher ME, McDermott CJ et al: Mutation analysis of the spastin gene (SPG4) in patients with hereditary spastic paraparesis. J Med Genet 2000; 37: 759-765.

11 Patrono C, Scarano V, Cricchi F et al: Autosomal dominant hereditary spastic paraplegia: DHPLC-based mutation analysis of SPG4 reveals eleven novel mutations. Hum Mutat 2005; 25: 506.

12 Sauter S, Miterski B, Klimpe S et al: Mutation analysis of the spastin gene (SPG4) in patients in Germany with autosomal dominant hereditary spastic paraplegia. Hum Mutat 2002; 20: $127-132$.

13 Crippa F, Panzeri C, Martinuzzi A et al: Eight novel mutations in SPG4 in a large sample of patients with hereditary spastic paraplegia. Arch Neurol 2006; 63: 750-755.

14 Depienne C, Tallaksen C, Lephay JY et al: Spastin mutations are frequent in sporadic spastic paraparesis and their spectrum is different from that observed in familial cases. J Med Genet 2006; 43: $259-265$.

15 Confalonieri F, Duguet M: A 200-amino acid ATPase module in search of a basic function. Bioessays 1995; 17: 639-650.

16 Frickey T, Lupas AN: Phylogenetic analysis of AAA proteins. J Struct Biol 2004; 146: 2-10.

17 Patel S, Latterich M: The AAA team: related ATPases with diverse functions. Trends Cell Biol 1998; 8: 65-71.

18 Hanson PI, Whiteheart SW: AAA+ proteins: have engine, will work. Nat Rev Mol Cell Biol 2005; 6: 519-529.

19 Beetz C, Nygren AO, Schickel J et al: High frequency of partial SPAST deletions in autosomal dominant hereditary spastic paraplegia. Neurology 2006; 67: 1926-1930.

20 Depienne C, Fedirko E, Forlani S et al: Exon deletions of SPG4 are a frequent cause of hereditary spastic paraplegia. J Med Genet 2007; 44: 281-284.

21 Harding AE: Classification of the hereditary ataxias and paraplegias. Lancet 1983; 1: 1151-1155.

22 Pantakani DV, Swapna LS, Srinivasan N, Mannan AU: Spastin oligomerizes into a hexamer and the mutant spastin (E442Q) redistribute the wild-type spastin into filamentous microtubule. J Neurochem 2008; 106: 613-624.

23 Meijer IA, Hand CK, Cossette P et al: Spectrum of SPG4 mutations in a large collection of North American families with hereditary spastic paraplegia. Arch Neurol 2002; 59: 281-286. 
24 Schickel J, Beetz C, Frommel C et al: Unexpected pathogenic mechanism of a novel mutation in the coding sequence of SPG4 (spastin). Neurology 2006; 66: 421-423.

25 Svenson IK, Ashley-Koch AE, Gaskell PC et al: Identification and expression analysis of spastin gene mutations in hereditary spastic paraplegia. Am J Hum Genet 2001; 68: 1077-1085.

26 Mead SH, Proukakis C, Wood N et al: A large family with hereditary spastic paraparesis due to a frame shift mutation of the spastin (SPG4) gene: association with multiple sclerosis in two affected siblings and epilepsy in other affected family members. J Neurol Neurosurg Psychiatry 2001; 71: 788-791.

27 Falco M, Scuderi C, Musumeci S et al: Two novel mutations in the spastin gene (SPG4) found by DHPLC mutation analysis. Neuromuscul Disord 2004; 14: 750-753.

28 Park SY, Ki CS, Kim HJ et al: Mutation analysis of SPG4 and SPG3A genes and its implication in molecular diagnosis of Korean patients with hereditary spastic paraplegia. Arch Neurol 2005; 62: $1118-1121$.

29 Beetz C, Zuchner S, Ashley-Koch A et al: Linkage to a known gene but no mutation identified: comprehensive reanalysis of SPG4 HSP pedigrees reveals large deletions as the sole cause. Hum Mutat 2007; 28: 739-740.

30 White SR, Evans KJ, Lary J, Cole JL, Lauring B: Recognition of C-terminal amino acids in tubulin by pore loops in Spastin is important for microtubule severing. J Cell Biol 2007; 176: $995-1005$.

31 Svenson IK, Kloos MT, Gaskell PC et al: Intragenic modifiers of hereditary spastic paraplegia due to spastin gene mutations. Neurogenetics 2004; 5: 157-164.

32 McDermott CJ, Burness CE, Rao Kirby J, Shaw PJ: Further insights into HSP and spastin: severe complicated phenotypes and evidence of lower motor neurone dysfunction (Abstract number 059); Proceedings of Clinical Neurosciences, Torquay, Devon, 7-9 September 2005; neurology abstracts only. I Neurol Neurosurg Psychiatry 2006; 77: 126-142.

33 Nielsen JE, Koefoed P, Kjaergaard S, Jensen LN, Nørremølle A, Hasholt L: Prenatal diagnosis of autosomal dominant hereditary spastic paraplegia (SPG4) using direct mutation detection. Prenat Diagn 2004; 24: 363-366.

34 Zhao GH, Tang BS, Luo W et al: Spastin gene mutation in Chinese patients with hereditary spastic paraplegia. Zhonghua Yi Xue Yi Chuan Xue Za Zhi 2003; 20: 177-180. (article in Chinese).

35 Errico A, Ballabio A, Rugarli EI: Spastin, the protein mutated in autosomal dominant hereditary spastic paraplegia, is involved in microtubule dynamics. Hum Mol Genet 2002; 11: 153-163.

36 Evans KJ, Gomes ER, Reisenweber SM, Gundersen GG, Lauring BP: Linking axonal degeneration to microtubule remodeling by Spastin-mediated microtubule severing. J Cell Biol 2005; 168: 599-606.

37 Chinnery PF, Keers SM, Holden MJ, Ramesh V, Dalton A: Infantile hereditary spastic paraparesis due to codominant mutations in the spastin gene. Neurology 2004; 63: 710-712.

38 Yip AG, Durr A, Marchuk DA et al: Meta-analysis of age at onset in spastin-associated hereditary spastic paraplegia provides no evidence for a correlation with mutational class. $J$ Med Genet 2003; 40: e106.

39 Harding AE: Hereditary 'pure' spastic paraplegia: a clinical and genetic study of 22 families. J Neurol Neurosurg Psychiatry 1981; 44: $871-883$.

40 Ivanova N, Löfgren A, Tournev I et al: Spastin gene mutations in Bulgarian patients with hereditary spastic paraplegia. Clin Genet 2006; 70: 490-495.

Supplementary Information accompanies the paper on European Journal of Human Genetics website (http://www.nature.com/ejhg) 